\title{
LA PARTICIPACIÓN SOCIAL ORGANIZADA EN LA LUCHA CONTRA LA POBREZA: la experiencia del Pronasol en tRes ESTADOS DEL NORTE DE MÉXICO
}

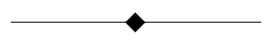

\begin{abstract}
RESUMEN
El objetivo de este trabajo es presentar, por medio del análisis de información empírica referente a tres estados del norte de México, una aproximación al estudio de las organizaciones vecinales que se incorporaron o surgieron al amparo del Programa Nacional de Solidaridad (Pronasol) con el propósito de coadyuvar a las tareas involucradas en la puesta en marcha y operación de proyectos de beneficio colectivo. Dada la envergadura que llegaron a alcanzar estas organizaciones en la estrategia del programa y en el ámbito social, diversos analistas de la época comenzaron a poner en duda los supuestos que justificaban la constitución de los Comités de Solidaridad, argumentando sus posibles nexos con esquemas de control neocorporativo o aduciendo sus limitaciones para superar una forma de participación meramente instrumental. Independientemente de sus peculiaridades, en el fondo estas posiciones críticas coincidían con una hipótesis general: la participación organizada en el marco de Solidaridad no representa una alternativa de cambio efectiva en los procesos de reforma democrática del Estado mexicano.

En este trabajo se pretenden explorar algunas respuestas a la hipótesis anterior, dividiendo a los Comités en dos planos: como instancias de participación y gestión comunitaria, y como organizaciones vinculadas con posibles esquemas de control corporativo.

Palabras clave: política, pobreza, participación, organización, bienestar.
\end{abstract}

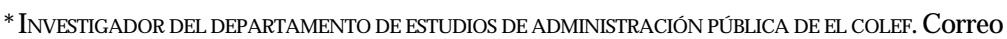
electrónico: ordonezbarba@hotmail.com 


\section{ABSTRACT}

The aim of this work is to show, through the analysis of empirical data of three states of the north of Mexico, an approximation to the study of neighbour organizations incorporated or originated by the shelter of the Solidarity $\mathrm{N}$ ational Program (Pronasol) in order to cooperate in the tasks related with theapplication and operation of projects for collective benefit.

Considering the importance this organizations obtained in the strategy of the program and in the social context, different analysts of that time began to doubt about the arguments that justified the creation of the Solidarity Committees, arguing possible bonds with neocorporative control plans or noticing their limitations for overcoming a merely instrumental participation. Independently of their peculiarities, this critics coincided in a general hypothesis: organised participation in the context of the Solidarity Program doesn't represent an effective alternative for a change in the process for a democratic reform of the Mexican State.

In the present paper, we pretend to explore some answers to that hypothesis dividing Committees in two levels: as instances of communitary participation and management, and as organisations linked with possible schemes of corporative control.

Keywords: policy, poverty, participation, organization, welfare.

\section{INTRODUCCIÓN}

A raíz dela crisis económica de 1982, el Estado mexicano emprendió varios procesos de reforma con el fin deracionalizar las políticas de bienestar en diversos sentidos, pero con el propósito común deajustar su expansión y prioridades a las posi bilidades financieras dela federación, delos gobiernos locales y desus propios beneficiarios. En general, estos cambios setradujeron en nuevas barreras de acceso a los servicios sociales el emental es, sobre todo para los más pobres. En estemarco, desdeel inicio desu sexenio, Salinas de Gortari (1988-1994) instaura el Programa Nacional deSolidaridad (Pronasol) como una iniciativa de lucha contra la pobreza con la misión decubrir, en al guna proporción, los huecos abiertos por el ajustegubernamental y el deterioro económico, tanto en el ámbito rural como en el urbano.

Una de las principales contribuciones de Solidaridad, según sus propios promotores, estribaría en su potencial para romper con las inercias que habían dominado hasta entonces las relaciones entreel Estado y la sociedad duranteel proceso de construcción y 
ampliación delas políticas debienestar. Deacuerdo con su concepción, a lo largo de la etapa previa las políticas social es habían tendido a estructurarse atendiendo más a las dinámicas de reproducción del poder en manos del ejecutivo federal, delas burocracias y delas corporaciones ligadas al partido en el gobierno, que a las propias necesidades sociales, dando como resultado un sistema dereparto injusto, costoso, central ista, burocratizado, corporativo y paternalista. Con el propósito de introducir orientaciones distintas en la formulación e implementación de las políticas, el programa propone nuevas bases no usadas antes para lograr un acercamiento efectivo entre las instituciones encargadas de la dotación debienes y servicios públicos y sus potenciales beneficiarios. Deacuerdo con el diseño, el éxito dela estrategia no sólo implicaba eliminar a los intermediarios tradicionales o descentralizar responsabilidades hacia los nivel es locales de gobierno, también exigía de la sociedad corresponsabilidad y participación frentea sus necesidades y frente a los responsables gubernamentales. En su lógica, ambos movimientos (uno descendentedesdelas instituciones y otro ascendente desde la óptica social) permitirían, además de acortar distancias en la relación gobierno-sociedad, mejorar la eficacia y rentabilidad delas acciones, reducir burocracias, eludir los canales corporativos y afianzar la construcción de una cultura ciudadana moderna y activa.

Con el fin de darlecontenido a la parte social de la ecuación, el programa se propuso al entar la formación de organizaciones vecinales basadas en un modelo quedenominaron Comité deSolidaridad y cuyo funcionamiento estaría regido por cuatro principios fundamentales: a) respeto a la voluntad, iniciativas y formas de organización de los individuos y las comunidades; b) participación plena y efectiva delas comunidades en todas las acciones del programa; c) corresponsabilidad entreel Estado y la sociedad organizada; y d) manejo honesto y transparentedelos recursos (Consejo Consultivo, 1994:60-62). Deacuerdo con el proyecto, por medio deestos Comités los grupos beneficiados podrían: realizar un ejercicio de democracia al elegir libremente en asamblea a sus representantes (o mesas directivas); priorizar necesidades colectivas; 
distribuir responsabilidades; gestionar demandas antelas autoridades correspondientes; determinar las aportaciones de sus miembros (en dinero, materiales o trabajo); administrar los recursos públicos asignados; y, coordinar las tareas de ejecución, control y fiscalización delas obras o acciones emprendidas.

Derivado desu perfil democrático, las decisiones en esta forma de organización debían tomarse demanera colectiva a través dela asamblea, la cual estaría integrada con todos los posibles beneficiarios. Además deel egir a los miembros dela mesa directiva, en la asamblea se debían decidir todos los aspectos rel ativos al proceso de gestión, desde la formulación de las demandas hasta la recepción delas obras, pasando por las fases del diseño del proyecto, la estructura del financiamiento y la ejecución delas acciones. En este marco, las responsabilidades de la mesa directiva estarían circunscritas a funciones de representación, coordinación y seguimiento, y con la obligación de informar en cada paso a la Asamblea de los resultados de las gestiones y los avances de la obra o el proyecto. Para llevar a cabo sus trabajos, los órganos de dirección debían conformarse con al menos un presidente, un secretario y un tesorero, así como por los vocales que fueran necesarios. En 1991, es decir, a dos años de iniciado el programa, se agregó al modelo original de la mesa directiva la figura de un vocal de Control y Vigilancia, que asumiría las funciones de una Contraloría Social con facultades para vigilar la aplicación delos recursos y el desempeño del propio Comité y de las instituciones involucradas, así como para canalizar las consultas, quejas y denuncias ante las autoridades correspondientes (Consejo Consultivo, 1994:63-67).

A esta forma devinculación con la sociedad seleconceptual izó como el método de trabajo de Solidaridad y en su espíritu buscaba convertir "la gestión delas obras públicas en procesos demovilización, organización y corresponsabilidad”, así como en un ejercicio de planeación participativa que contribuye a definir prioridades a partir de demandas colectivas determinadas en unidades deterritorio microrregionales o comunitarias (Consejo Consultivo, 1994:6769). En varios sentidos, el Pronasol puede ser considerado como una iniciativa innovadora en el campo del combatea la pobreza en 
México. Sin embargo, desdenuestro punto devista, el planteamiento que desarrolla acerca de la participación social organizada sobresale como una de sus principales contribuciones al acervo de las estrategias previamente implantadas (véase Ordóñez, 1997). A tendiendo exclusivamentea la concepción, se podría argumentar que para Solidaridad la actuación pública adquiere contenidos, sentido y transparencia sólo a partir del momento en el quelogra constituirse un Comité, o en otras palabras, cuando seidentifican "interlocutores sociales verdaderamenterepresentativos dela demanda y de la participación de las comunidades" (Consejo Consultivo, 1994:138). Esta afirmación resul ta sólo parcialmentecierta si consideramos quela realización deun conjunto amplio deobras (hospitales, carreteras o construcción de infraestructura de gran escala) no pasaban por las decisiones comunitarias. No obstante esta acotación al método de trabajo, está claro queel programa puso un fuerteempeño para apoyar la conformación deComités en todo el territorio, logrando crear, deacuerdo con el informefinal, al rededor de 150 mil (Consejo Consultivo, 1994:136).

Dada la envergadura que llegó a alcanzar este proyecto en la estrategia del programa y en el ámbito social, diversos anal istas de la época comenzaron a poner en duda los supuestos que justificaban la constitución delos Comités como vehículos para consolidar nuevas relaciones entre el Estado y la sociedad. Entre los críticos había quienes opinaban que con esta forma de organización, más que romper con el corporativismo tradicional, se buscaba encontrar nuevas rutas para ampliar las bases social es de apoyo en un esquema de control neocorporativo (Dresser, 1994) que, además, ayudaría a minar a las organizaciones independientes arraigadas en el territorio (Bartra, 1992:27-34). Desde otro punto devista, había quienes cuestionaban el al cance de la participación social que podría producirse a través delos Comités, aduciendo las limitaciones para transitar de una forma de participación fundada en la solución de los problemas concretos de la pobreza (perspectiva instrumental ), a otra que permita colocar a las comunidades como actores relevantes en el proceso político (o democrático) en un sentido amplio (Provencio, 1992:35-55). Estas interpretaciones de los 
acontecimientos en el fondo coinciden con una hipótesis general: la participación organizada en el marco de Solidaridad no representa una al ternativa decambio efectiva en los procesos de reforma democrática del Estado mexicano.

Sin duda, las aristas de análisis que pudieran desprenderse de esta aproximación serían casi tan amplias como la imaginación disciplinaria en las ciencias sociales lo permita; incluso podría recurrirse a preguntas basadas en especulaciones que cobraron relevancia en la opinión pública en esos años. ${ }^{1}$ No obstante las dificultades para indagar y más aún para evaluar todas las implicaciones deestetipo de partici pación, con la información empírica que disponemos es posi ble acercarse a al gunas respuestas a la hipótesis anterior dividiendo el análisis en dos planos: el instrumental y el político. El orden de exposición parte de la idea que es necesario, en primer lugar, dividir los Comités desde una perspectiva instrumental, es decir, en su desempeño como vías de colaboración social y como instancias de gestión de demandas comunitarias. En un segundo plano, se procederá a investigar el perfil político de estas organizaciones y sus vínculos con posibles esquemas decontrol corporativo. Finalmente, se explorarán algunos elementos que permitan discutir la influencia del Pronasol en los procesos democráticos.

Lainformación quesirvedebase para esteestudio se derivó del proyecto de investigación denominado "Impacto social del Programa Nacional de Solidaridad", que El Colegio de la Frontera Norterealizó durante el tercer trimestre de 1993 en los estados de Coahuila, Durango y Tamaulipas. Particularmente se utilizarán las bases de datos que surgieron de dos encuestas: una dirigida a

${ }^{1}$ Como sucedió cuando en el PRI fue transformado el viejo "sector popular", que tradicionalmente estuvo dominado por los sindicatos de trabajadores al servicio del Estado (FSTSE), en lo que se denominó "el movimiento territorial" (o UNE). Con este cambio hubo quienes afirmaron que ese partido se preparaba para albergar a los líderes surgidos o identificados en las colonias con la intervención del Pronasol. En el extremo de esta percepción se llegó a conjeturar la intención del presidente Salinas de apropiarse del PRI o, en última instancia, de crear su propio partido político, el de la Solidaridad. 
beneficiariosy otra levantada entremiembros delas mesas directivas delos Comités. Para llevar a cabo el análisis, seaplicarán técnicas estadísticas simples y se presentará la información en forma comparada para identificar los cambios en el perfil y desempeño de los Comités en los tres contextos regional es investigados.

Cabe destacar quesi bien el trabajo está referido a un momento histórico queaconteció hace más de 10 años, el método detrabajo que desarrolló el Pronasol ha sido integrado como un elemento central en la instrumentación de nuevas iniciativas federales y locales en el combatea la pobreza, lo cual permiteactualizar la pertinencia de su estudio y ponderar la vigencia dela partici pación social organizada y dela autogestión como estrategias para avanzar en la superación deproblemas colectivos.

\section{LOS COMITÉS COMO CANALES}

DE PARTICIPACIÓN SOCIAL ORGANIZADA

Para comenzar a analizar el desempeño delos Comités deSolidaridad es preciso mencionar antes que, en los contextos regionales investigados, una buena partedelos beneficiarios efectivos del programa provino deestratos depoblación cuyos ingresosfamiliares (cuadro 1) superaban los niveles que determinan una situación de pobreza (entre 58y $73 \%$, según el caso). Considerando esteindicador se podría afirmar que, en la mayoría de los casos, las organizaciones comunitarias surgen en un medio social distinto para el que originalmentefuediseñad o el Pronasol. Esta distorsión en la composición de los beneficiarios induce, desde una perspectiva conceptual, modificaciones en el comportamiento esperado de la población estudiada y su reconocimiento nos ayuda a explicar el o los tipos de colaboración el egidos por los beneficiarios, así como la calidad dela gestión que desarrollan en los Comités. Considerando sól o los estratos deingreso familiar, podríamos suponer quela probabilidad para que una persona opte por contribuir al financiamiento con aportaciones monetarias, en detrimento de la colaboración en especie o en trabajo, se incrementa a medida que aumentan los ingresos disponibles, pero si aceptamos además que 
CUADRO 1. Beneficiarios del Pronasol según el ingreso diario por residente en el hogar.

\begin{tabular}{|c|c|c|c|c|c|c|c|c|c|c|}
\hline \multirow[t]{2}{*}{ Entidad } & \multirow[t]{2}{*}{$\begin{array}{l}\text { Condición } \\
\text { de pobreza }\end{array}$} & \multicolumn{2}{|c|}{$\begin{array}{l}\text { Ingresos en el hogar en sal arios } \\
\text { mínimos diarios por residente }\end{array}$} & \multicolumn{4}{|c|}{$\begin{array}{l}\text { ¿Harecibido al gún beneficio } \\
\text { deSolidaridad? (\%) }\end{array}$} & \multicolumn{3}{|c|}{ Beneficiarios (\%) } \\
\hline & & Rangos deingreso & $\%$ & Sí & No & NR & Total & Total & A & Al \\
\hline Coahuila & $\begin{array}{l}\text { Extrema } \\
\text { Moderada } \\
\text { No pobreza }\end{array}$ & $\begin{array}{l}\text { Menos de } 0.20 \mathrm{~s} . \mathrm{m} . \\
\text { Entre } 0.21 \text { y } 0.40 \mathrm{~s} . \mathrm{m} . \\
\text { Entre } 0.41 \text { y } 0.60 \mathrm{s.m} . \\
\text { Entre } 0.61 \text { y } 0.80 \mathrm{s.m} . \\
\text { Entre } 0.81 \text { y } 1 \mathrm{s.m} . \\
\text { Más de } 1 \mathrm{s.m} . \\
\text { No sabía o no respondió } \\
\text { Total }\end{array}$ & $\begin{array}{r}9.1 \\
28.7 \\
18.2 \\
9.5 \\
7.4 \\
20.5 \\
6.7 \\
100.0\end{array}$ & $\begin{array}{l}57.8 \\
64.9 \\
63.0 \\
58.7 \\
66.5 \\
40.3 \\
55.3 \\
57.8\end{array}$ & $\begin{array}{l}42.2 \\
33.7 \\
36.0 \\
41.3 \\
33.5 \\
57.3 \\
42.7 \\
41.0\end{array}$ & $\begin{array}{l}2.4 \\
1.9 \\
1.2\end{array}$ & $\begin{array}{l}100.0 \\
100.0 \\
100.0 \\
100.0 \\
100.0 \\
100.0 \\
100.0 \\
100.0\end{array}$ & $\begin{array}{r}9.1 \\
32.3 \\
19.8 \\
9.6 \\
8.5 \\
14.3 \\
6.4 \\
100.0\end{array}$ & $\begin{array}{r}9.1 \\
41.4 \\
61.2 \\
70.8 \\
79.3 \\
93.6 \\
100.0\end{array}$ & $\begin{array}{r}9.7 \\
44.2 \\
65.3 \\
75.6 \\
84.7 \\
100.0\end{array}$ \\
\hline Durango & $\begin{array}{l}\text { Extrema } \\
\text { Moderada } \\
\text { No pobreza }\end{array}$ & $\begin{array}{l}\text { Menos de } 0.20 \mathrm{~s} . \mathrm{m} . \\
\text { Entre } 0.21 \text { y } 0.40 \mathrm{~s} . \mathrm{m} . \\
\text { Entre } 0.41 \text { y } 0.60 \mathrm{~s} . \mathrm{m} . \\
\text { Entre } 0.61 \text { y } 0.80 \mathrm{~s} . \mathrm{m} . \\
\text { Entre } 0.81 \text { y } 1 \mathrm{s.m} . \\
\text { Más de } 1 \mathrm{s.m} \text {. } \\
\text { No sabía o no respondió } \\
\text { Total } \\
\end{array}$ & $\begin{array}{r}10.6 \\
23.9 \\
17.2 \\
6.8 \\
6.3 \\
16.8 \\
18.4 \\
100.0 \\
\end{array}$ & $\begin{array}{l}79.5 \\
86.5 \\
97.0 \\
78.8 \\
73.3 \\
82.8 \\
82.4 \\
84.8 \\
\end{array}$ & $\begin{array}{r}18.2 \\
12.3 \\
1.6 \\
21.2 \\
26.7 \\
14.3 \\
12.1 \\
12.9 \\
\end{array}$ & $\begin{array}{l}2.2 \\
1.1 \\
1.4 \\
\\
2.8 \\
5.6 \\
2.2 \\
\end{array}$ & $\begin{array}{l}100.0 \\
100.0 \\
100.0 \\
100.0 \\
100.0 \\
100.0 \\
100.0 \\
100.0 \\
\end{array}$ & $\begin{array}{r}9.9 \\
24.4 \\
19.7 \\
6.4 \\
5.4 \\
16.4 \\
17.8 \\
100.0 \\
\end{array}$ & $\begin{array}{r}9.9 \\
34.3 \\
54.0 \\
60.4 \\
65.8 \\
82.2 \\
100.0\end{array}$ & $\begin{array}{r}12.0 \\
41.7 \\
65.7 \\
73.5 \\
80.1 \\
100.0\end{array}$ \\
\hline Tamaulipas & $\begin{array}{l}\text { Extrema } \\
\text { Moderada } \\
\text { No pobreza }\end{array}$ & $\begin{array}{l}\text { Menos de } 0.20 \mathrm{~s} . \mathrm{m} . \\
\text { Entre } 0.21 \text { y } 0.40 \mathrm{~s} . \mathrm{m} . \\
\text { Entre } 0.41 \text { y } 0.60 \mathrm{~s} . \mathrm{m} . \\
\text { Entre } 0.61 \text { y } 0.80 \mathrm{~s} . \mathrm{m} . \\
\text { Entre } 0.81 \text { y } 1 \mathrm{s.m} . \\
\text { Más de } 1 \mathrm{s.m} \text {. } \\
\text { No sabía o no respondió } \\
\text { Total } \\
\end{array}$ & $\begin{array}{r}6.5 \\
18.1 \\
21.6 \\
13.3 \\
7.0 \\
28.5 \\
5.2 \\
100.0\end{array}$ & $\begin{array}{l}43.5 \\
69.3 \\
61.2 \\
51.5 \\
57.1 \\
66.5 \\
48.5 \\
60.8 \\
\end{array}$ & $\begin{array}{l}56.5 \\
30.0 \\
38.8 \\
47.8 \\
42.9 \\
33.5 \\
36.7 \\
38.2\end{array}$ & $\begin{array}{r}0.7 \\
0.7 \\
14.8 \\
1.0 \\
\end{array}$ & $\begin{array}{l}100.0 \\
100.0 \\
100.0 \\
100.0 \\
100.0 \\
100.0 \\
100.0 \\
100.0\end{array}$ & $\begin{array}{r}4.6 \\
20.6 \\
21.7 \\
11.2 \\
6.5 \\
31.2 \\
4.2 \\
100.0\end{array}$ & $\begin{array}{r}4.6 \\
25.2 \\
46.9 \\
58.1 \\
64.6 \\
95.8 \\
100.0\end{array}$ & $\begin{array}{r}4.8 \\
26.3 \\
48.9 \\
60.6 \\
67.4 \\
100.0\end{array}$ \\
\hline Totales & $\begin{array}{l}\text { Extrema } \\
\text { Moderada } \\
\text { No pobreza }\end{array}$ & $\begin{array}{l}\text { Menos de } 0.20 \mathrm{~s} . \mathrm{m} . \\
\text { Entre } 0.21 \text { y } 0.40 \mathrm{~s} . \mathrm{m} . \\
\text { Entre } 0.41 \text { y } 0.60 \mathrm{s.m} . \\
\text { Entre } 0.61 \text { y } 0.80 \mathrm{s.m} . \\
\text { Entre } 0.81 \text { y } 1 \mathrm{s.m} . \\
\text { Más de } 1 \mathrm{s.m} . \\
\text { No sabía o no respondió } \\
\text { Total }\end{array}$ & $\begin{array}{r}8.5 \\
23.8 \\
19.2 \\
10.3 \\
7.0 \\
22.6 \\
8.7 \\
100.0\end{array}$ & $\begin{array}{l}59.7 \\
70.9 \\
68.8 \\
58.2 \\
64.4 \\
59.2 \\
66.2 \\
64.7\end{array}$ & $\begin{array}{l}39.7 \\
28.0 \\
30.5 \\
41.5 \\
35.6 \\
39.4 \\
27.3 \\
33.9\end{array}$ & $\begin{array}{l}0.6 \\
1.1 \\
0.7 \\
0.3 \\
1.4 \\
6.4 \\
1.4\end{array}$ & $\begin{array}{l}100.0 \\
100.0 \\
100.0 \\
100.0 \\
100.0 \\
100.0 \\
100.0 \\
100.0\end{array}$ & $\begin{array}{r}7.8 \\
26.0 \\
20.4 \\
9.2 \\
6.9 \\
20.7 \\
9.0 \\
100.0\end{array}$ & $\begin{array}{r}7.8 \\
33.8 \\
54.2 \\
63.4 \\
70.3 \\
91.0 \\
100.0\end{array}$ & $\begin{array}{r}8.6 \\
37.2 \\
59.6 \\
69.7 \\
77.3 \\
100.0\end{array}$ \\
\hline
\end{tabular}

NotAs: NR: No respuesta; A: Porcentaje acumulado; AI: Porcentaje acumulado entre las familias con ingresos conocidos. Fuente: El Colef, Proyecto: Impacto social del Programa Nacional de Solidaridad en los estados de Coahuila, Durango y Tamaulipas, tercer trimestre de 1993. "Cédula para la identificación de beneficiarios." 
esteindicador es sólo una característica que denota mejores niveles de bienestar (en educación, salud, alimentación, etcétera) podríamos esperar que también aumente la capacidad para lograr una participación demejor calidad en el seguimiento y desarrollo delas tareas col ectivas. La confirmación o rechazo de estos escenarios en cual quier contexto social requeriría de una investigación mucho más profunda de la que nos hemos propuesto; sin embargo, a través de los casos estudiad os es posi bleobservar al gunas tendencias que del inean la trayectoria aguardada.

Con la información disponible (cuadro 2) podemos observar que, en general, una gran parte dela población beneficiaria mostró amplia disposición para contribuir de diversas maneras a la realización de los proyectos colectivos. Los datos indican que al rededor dela mitad delas familias beneficiadas con obras o acciones realizadas en su entorno inmediato (comunidades o col onias) ofreció alguna forma de apoyo. Según los tipos decolaboración seadvierte que, de este subconjunto, un poco más de la mitad ayudaron al financiamiento de las obras a través deaportaciones en dinero (55\%) o en trabajo (52\%), casi $80 \%$ acudieron a por lo menos una reunión devecinos y más deuna cuarta partedeellos (27\%) seincorporó en al guna comisión detrabajo. El hecho de quela gran mayoría deesta población participantesupiera dela existencia delos Comités (88\%) y quemás de $54 \%$ deellos haya presenciado la el ección dela mesa directiva, confirma quetales niveles departicipación searticularon medianteestas organizaciones. En principio, esta evidencia sugierequeel programa logró despertar el interés de sus clientelas por participar y estructurarseen torno suyo y por medio desu modelo de organización.

Desdeun punto devista comparativo, no obstantequeen los tres casos estudiados se perfila un contexto de movilización social de importantes dimensiones, los datos revelan la presencia de diferencias significativas en los grados y tipos de participación alcanzados en cada entidad. En el extremo inferior encontramos queen la entidad deDurango, el estado económicamente más débil delos tres y en el cual el programa obtuvo un grado deerror en la selección de beneficiarios menor al promedio, la proporción de población 
CUADRO 2. Participación de los beneficiarios de Solidaridad en las comunidades donde se ejecutaron obras o se llevaron a cabo acciones específicas del programa (\% sólo de respuestas afirmativas).

\begin{tabular}{|c|c|c|c|c|c|}
\hline \multicolumn{2}{|c|}{ Indicadores de participación social } & Coahuila & Durango & Tamaulipas & Total \\
\hline \multicolumn{2}{|c|}{ En su colonia existe al guna obra o acción de Solidaridad } & 70.2 & 71.9 & 71.5 & 71.1 \\
\hline \multicolumn{2}{|c|}{ En esa obra participó usted } & 53.4 & 44.9 & 59.5 & 53.1 \\
\hline \multirow[t]{5}{*}{ Dequémanera participó: } & A portando dinero & 55.8 & 39.9 & 64.6 & 55.4 \\
\hline & A portando trabajo & 51.7 & 74.0 & 38.9 & 52.1 \\
\hline & A portando materiales & 8.2 & 22.8 & 7.7 & 11.6 \\
\hline & Asistiendo a alguna reunión & 80.7 & 81.2 & 69.3 & 76.4 \\
\hline & Formando partedeal guna comisión & 35.7 & 31.1 & 17.2 & 27.4 \\
\hline \multicolumn{2}{|c|}{ Ha oído hablar del Comité de Solidaridad } & 87.6 & 87.4 & 90.2 & 88.6 \\
\hline \multicolumn{2}{|c|}{ Estuvo presenteen la elección del Comité } & 52.6 & 53.1 & 56.9 & 54.4 \\
\hline \multirow{6}{*}{$\begin{array}{l}\text { En quéforma seeligió } \\
\text { el Comité: } \\
\text { (Respuestas excluyentes) }\end{array}$} & En una asamblea & 63.9 & 86.7 & 80.0 & 75.9 \\
\hline & En una elección por voto secreto & 14.9 & 0.0 & 5.7 & 7.6 \\
\hline & Nombramiento de promotores de Solidaridad & 3.2 & 5.6 & 1.5 & 3.0 \\
\hline & Por una comisión decolonos & 14.8 & 5.8 & 9.0 & 10.3 \\
\hline & Nombramiento deautoridades municipales & 0.9 & 0.0 & 2.2 & 1.2 \\
\hline & Nombramiento deautoridades estatales & 0.0 & 1.9 & 1.1 & 0.8 \\
\hline \multirow{3}{*}{\multicolumn{2}{|c|}{$\begin{array}{l}\text { Ocupa usted al gún cargo en el Comité } \\
\text { El Comitérepresenta los intereses dela comunidad } \\
\text { El Comitépertenecea al gún partido político }\end{array}$}} & 19.1 & 19.3 & 15.2 & 17.7 \\
\hline & & 78.6 & 86.2 & 80.0 & 81.0 \\
\hline & & 44.7 & 43.7 & 43.6 & 44.0 \\
\hline \multirow[t]{4}{*}{ A cuál partido: } & PRI & 88.9 & 79.2 & 98.8 & 90.4 \\
\hline & PAN & 2.3 & 4.6 & 1.2 & 2.4 \\
\hline & PRD & 3.1 & 0.0 & 0.0 & 1.1 \\
\hline & Otros & 1.5 & 16.3 & 0.0 & 4.5 \\
\hline
\end{tabular}

Fuente: El Colef, Proyecto: Impacto social del Programa Nacional de Solidaridad en los estados de Coahuila, Durango y Tamaulipas, tercer trimestre de 1993. “Cédula para la identificación de beneficiarios" y “Cuestionario a beneficiarios". 
beneficiada participante fue la más baja y en su mayoría optaron por formas decolaboración basadas en aportaciones en trabajo. En el punto superior del espectro tenemos que en Tamaulipas, una entidad con una economía menos vulnerabley dondeel Pronasol obtuvo el peor desempeño en la identificación desu población objetivo, los beneficiarios manifestaron los niveles más al tos de participación y entre el los la mayoría tuvo la posibilidad de financiar los proyectos con recursos monetarios, pero en contraparte se observó menos interés por asumir otras responsabilidades, como acudir a las reuniones o formar partedeal guna comisión.

Con el estudio de estos dos casos, y tomando en cuenta que el tercero (es decir, Coahuila) se ajusta a lo esperado, se puede establecer queexisten correlaciones positivas entrelos niveles deingreso familiar y el alcance y las formas de la colaboración social enmarcados en el modelo de Solidaridad. En general, las tendencias indican que a mayor pobreza habrá menos probabilidad para queun conjunto deindividuos o familias participe, pero cuando lo hace ofrece preferentemente su trabajo para la realización de las obras y muestra mayor disposición para informarsey colaborar en tareas de gestión. Por el contrario, en comunidades quedisponen de más recursos la movilización social es más amplia y con mayores posibilidades para financiar con dinero las obras de interés colectivo, pero en este caso la participación tiende a reducirse en asuntos relacionados con la organización.

Independientementedelas divergencias en el comportamiento de la participación social en cada entidad, seobserva en todos los casos quelos Comités gozaron deamplio reconocimiento entrelos beneficiarios como agrupaciones representativas de los intereses dela comunidad (en promedio, $81 \%$ de quienes sabían desu existencia). La confianza que despertaron estas organizaciones parece estar vinculada a los métodos empleados en la elección de los re presentantes (en asambleas o en elecciones secretas contra otros mecanismos) y ser independientedela filiación desus miembrosa un partido político. Un poco más adelante analizaremos con más detalle los elementos asociados con cuestiones políticas, pero en estemomento resul ta evidentequelas prácticas democráticas en la 
formación delos Comités permitieron aumentar su aceptación en las comunidades y ayudaron a incentivar los esfuerzos decolaboración individual o familiar puestos en favor de los proyectos colectivos.

\section{LOS COMITÉS COMO INSTANCIAS DE GESTIÓN}

Otros factores que pueden ayudar a explicar la confianza quegeneraron los Comités como articuladores de la participación en sus comunidades están asociados al trabajo que desarrollaron en el proceso degestión y ejecución delos proyectos. A proximándonos en esta dirección, es factibleafirmar queotra gran fuentedela legitimidad captada por estas organizaciones sefincó en el compromiso quemostraron sus órganos de dirección en la realización delas tareas ejecutivas y en el seguimiento de las gestiones hasta la conclusión de las obras. Como puede apreciarse en los dos cuadros siguientes, la disposición delas mesas directivas a contribuir como unidades operativas del programa abarcó toda la gama deresponsabilidades y actividades técnicas requeridas para la operación de los proyectos (cuadro 3), permitiéndoles al final obtener buenos resultados como entidades financieras y ejecutoras de las acciones emprendidas (cuadro 4). Desdeesta perspectiva, las distancias que se observan entre los casos estudiados sugieren la existencia de una relación estrecha entre la capacidad de una sociedad local para organizarse y sus probabilidades de obtener éxito en la gestión de demandas colectivas.

En este punto, los orígenes delas divergencias que muestran los extremos (nuevamenteentreTamaulipas y Durango) sin duda van más al lá de los aspectos rel acionados con el perfil socioeconómico delas comunidades, debido a la incorporación de factores ajenosa su capacidad de decisión o a el ementos asociados a la gestión de subprogramas que requieren de una menor participación organizada. Estas dimensiones bien pudieran explicar una parte de lo quesucedió en Durango, tomando en cuenta queahí al rededor de una cuarta parte de los proyectos habían sido rechazados o aun se encontraban en espera deaprobación y casi una quinta partedelos 
Comi tés estaban orientados sól o a obtener financiamientos para la producción en el campo. No obstantelo anterior, los datos obligan a ponderar la importancia de un medio social menos precario como espacio para el surgimiento de organizaciones comunitarias mejor estructuradas, con mayores recursos e influencias y más capaces para culminar satisfactoriamente sus cometidos. En este sentido también habría que reconocer queen Durango el predominio delo rural (los ejidos, uno de los sectores más rezagados dela sociedad mexicana) entrelas comunidades beneficiadas pudo haber afectado el desempeño desus organizaciones en la gestión delas obras y constituirse en una condicionante que limitó las posi bilidades de sus mi embros para aportar dinero a los proyectos.

Con la conclusión anterior no estamos sugiriendo queSolidaridad debió modificar su objetivo central (es decir, combatir la indigencia en todos sus ámbitos y manifestaciones sociales) o, en su defecto, eliminar las restricciones de las acciones públicas que re quirieran de una intensa participación social organizada. Por el contrario, los resultados muestran quela eficacia global deun programa de esta naturaleza depende de ambas dimensiones: por un lado, dela aplicación de criterios más rigurosos en la selección de beneficiariosy, por el otro, de poner mayor énfasis en actividades queayuden a el evar la capaci dad deautogestión en las comunidades. El estudio de las experiencias estatales demuestra que la autogestión efectivamente puede ser una alternativa para incrementar la rentabilidad delas inversiones públicas en el territorio, pero para llevarla a cabo en condiciones de alta focalización es recomendable ampliar las oportunidades de capacitación y asistencia técnica en el diseño y en los procesos de administración, control y ejecución deproyectos.

\section{Los Comités DESDE UnA PERSPECTIVA POLÍTICA}

Antes deiniciar con el estudio dela dimensión política, cabeprecisar quelos Comités deSolidaridad se crean al amparo dela intervención pública y, en ese sentido, sus características dependen en gran medida dela actuación delosintermediarios del programa en 
CuAdRo 3. Indicadores de gestión y representatividad delos Comités de Solidaridad (\%).

\begin{tabular}{|c|c|c|c|c|c|c|c|c|c|}
\hline \multirow{2}{*}{\multicolumn{2}{|c|}{$\begin{array}{l}\text { Indicadores degestión y actividades real izadas } \\
\text { El Comitéen el queusted participa cuenta con: }\end{array}$}} & \multicolumn{2}{|c|}{ Coahuila } & \multicolumn{2}{|c|}{ Durango } & \multicolumn{2}{|c|}{ Tamaulipas } & \multicolumn{2}{|c|}{ Total } \\
\hline & & TR & Sí & TR & Sí & TR & Sí & TR & Sí \\
\hline \multirow{4}{*}{\multicolumn{2}{|c|}{$\begin{array}{l}\text { Un programa de trabajo } \\
\text { Un archivo con la documentación dela obra } \\
\text { Constancias delas decisiones tomadas en el Comité } \\
\text { Control sobre los costos dela obra }\end{array}$}} & 78.5 & 50.2 & 66.4 & 32.8 & 81.3 & 55.5 & 76.1 & 47.9 \\
\hline & & 78.1 & 80.8 & 67.3 & 46.3 & 81.7 & 84.9 & 76.3 & 74.0 \\
\hline & & 77.9 & 75.9 & 65.8 & 46.6 & 82.1 & 76.7 & 75.9 & 69.4 \\
\hline & & 77.2 & 83.4 & 67.8 & 41.6 & 84.7 & 83.9 & 77.0 & 73.8 \\
\hline \multicolumn{10}{|c|}{ Durantela ejecución dela obra el Comité: } \\
\hline \multirow{5}{*}{\multicolumn{2}{|c|}{$\begin{array}{l}\text { Organizó la aportación de la comunidad en trabajo } \\
\text { Recabó la aportación dela comunidad en dinero } \\
\text { Llevó un control delas aportaciones } \\
\text { Consiguió al contratista para la construcción de la obra } \\
\text { Compró los materiales para la realización del proyecto }\end{array}$}} & 80.6 & 47.7 & 72.8 & 55.8 & 85.6 & 57.7 & 80.0 & 53.0 \\
\hline & & 81.0 & 86.1 & 70.1 & 50.3 & 86.8 & 73.0 & 80.1 & 73.3 \\
\hline & & 81.0 & 79.9 & 71.3 & 55.6 & 84.2 & 79.3 & 79.4 & 73.9 \\
\hline & & 80.4 & 51.4 & 70.3 & 31.0 & 80.8 & 64.7 & 77.8 & 50.8 \\
\hline & & 80.0 & 43.4 & 70.3 & 24.6 & 82.6 & 57.7 & 78.2 & 43.6 \\
\hline \multirow{7}{*}{$\begin{array}{l}\text { Representatividad } \\
\text { delos Comités: }\end{array}$} & Varias colonias & & 6.0 & & 2.0 & & 5.0 & & 5.0 \\
\hline & Una colonia & & 34.0 & & 9.0 & & 27.0 & & 25.0 \\
\hline & Una parte dela colonia & & 30.0 & & 13.6 & & 11.0 & & 20.0 \\
\hline & Una escuela & & 23.0 & & 12.0 & & 33.0 & & 23.0 \\
\hline & Un ejido & & 3.0 & & 57.0 & & 22.0 & & 23.0 \\
\hline & Una organización deproductores & & 0.4 & & 1.0 & & 0.0 & & 0.5 \\
\hline & Otro & & 3.0 & & 2.0 & & 1.0 & & 2.0 \\
\hline
\end{tabular}

CódIGos: TR = Tasa de respuesta. La TR se refiere a la proporción de entrevistados que respondieron afirmativa o negativamente a las preguntas o, en otras palabras, excluye a quienes no ofrecieron respuesta o dijeron no saber del asunto.

FuENTE: El Colef, Proyecto: Impacto social del Programa Nacional de Solidaridad en los estados de Coahuila, Durango y Tamaulipas, tercer trimestre de 1993. "Cuestionario a miembros de Comités de Solidaridad." 
CUADRO 4. Situación de la última obra gestionada por los Comités de Solidaridad según subprogramas.

\begin{tabular}{|c|c|c|c|c|c|c|c|c|c|c|c|c|c|c|c|c|c|}
\hline \multicolumn{2}{|c|}{ Subprogramas } & \multicolumn{6}{|c|}{ Situación delaobra (\%) } & \multicolumn{2}{|c|}{$\begin{array}{l}\text { Costo } \\
\text { medio }\end{array}$} & \multicolumn{2}{|c|}{$\begin{array}{l}\text { Aportación } \\
\text { comunitaria }\end{array}$} & \multicolumn{6}{|c|}{ Aportación comunitariaen (\%): } \\
\hline \multirow[t]{2}{*}{ Tipos } & \multirow[t]{2}{*}{$\%$} & \multirow{2}{*}{$\begin{array}{l}\text { Recha } \\
\text { zada }\end{array}$} & \multirow{2}{*}{$\begin{array}{l}\text { Sinde- } \\
\text { disión }\end{array}$} & \multirow{2}{*}{$\begin{array}{l}\text { Apro- } \\
\text { bada }\end{array}$} & \multirow{2}{*}{$\begin{array}{l}\text { En pro- } \\
\text { ceso }\end{array}$} & \multirow{2}{*}{$\begin{array}{l}\text { Internum- } \\
\text { pida }\end{array}$} & \multirow{2}{*}{$\begin{array}{l}\text { Condui- } \\
\text { da }\end{array}$} & \multirow[t]{2}{*}{ TR } & \multirow[t]{2}{*}{$\mathbf{m} \$$} & \multirow[t]{2}{*}{ TR } & \multirow[t]{2}{*}{$\%$} & \multicolumn{2}{|c|}{ Dinero } & \multicolumn{2}{|c|}{ Materiales } & \multicolumn{2}{|c|}{ Trabajo } \\
\hline & & & & & & & & & & & & TR & Sí & TR & Sí & TR & Sí \\
\hline Total & 100 & 2 & 13 & 8 & 11 & 4 & 57 & 60 & 40.2 & 64 & 32 & 67 & 83 & 54 & 12 & 61 & 45 \\
\hline Escuela digna & 30 & 2 & 5 & 11 & 7 & 2 & 72 & 86 & 25 & 85 & & 86 & 80 & 4 & 14 & 83 & 44 \\
\hline / drenaje & 20 & 3 & 15 & 5 & 18 & 2 & 5 & 66 & 57 & 77 & & 72 & 2 & 4 & 5 & 65 & 41 \\
\hline Electrificación & 8 & 7 & 18 & 3 & 11 & 5 & 5 & 46 & 51 & 62 & 3 & 67 & 88 & 8 & 10 & 1 & 35 \\
\hline Infraestructura & 29 & 1 & 20 & 9 & 10 & 7 & 5 & 47 & & 53 & & 62 & 82 & 49 & 14 & 6 & 54 \\
\hline Producción & 8 & & 11 & 5 & 19 & 5 & 56 & 34 & 16.7 & 27 & 33 & 37 & 65 & 31 & 16 & 34 & 43 \\
\hline Coahuila & 100 & 2 & 13 & 5 & 8 & 3 & 64 & 64 & 28.4 & 69 & 28 & 77 & 94 & 63 & 9 & 65 & 29 \\
\hline Escue & 27 & 2 & - & 7 & 2 & & 8 & 94 & 21 & 9 & 2 & 98 & 99 & o & 9 & 84 & 18 \\
\hline Agua/ & 21 & 3 & 9 & 3 & 15 & & 67 & 66 & 21 & 81 & 30 & 85 & 96 & 70 & 4 & 72 & 27 \\
\hline Electrificación & 10 & & 16 & & 12 & 9 & 62 & 44 & 38.5 & 62 & 3 & 72 & 87 & 3 & 6 & 56 & 28 \\
\hline Infraestructura & 33 & 2 & 21 & 6 & 9 & 3 & 58 & 50 & 38.8 & 52 & 28 & 65 & 90 & 53 & 14 & 56 & 47 \\
\hline Producción & 5 & & 25 & 6 & 6 & 19 & 44 & 75 & 20.2 & 69 & 29 & 75 & 92 & 63 & 10 & 63 & 10 \\
\hline Durango & 100 & 1 & 23 & 8 & 14 & 6 & 40 & 38 & 35.2 & 32 & 20 & 41 & 57 & 35 & 18 & 47 & 71 \\
\hline Escuela digna & 22 & 2 & 13 & 24 & 7 & 9 & 44 & 73 & 32.8 & 5 & 22 & 73 & 58 & 64 & 21 & 76 & 56 \\
\hline Agua/ drenaje & 17 & 6 & 40 & 3 & 20 & 3 & 29 & 49 & 44.4 & 43 & 19 & 40 & 64 & 29 & 10 & 43 & 80 \\
\hline Electrificación & 4 & & 67 & & 11 & & 11 & 55 & 8.9 & 33 & 25 & 44 & 50 & 44 & 25 & 44 & 75 \\
\hline Infraestructura & 31 & & 27 & 5 & 11 & 11 & 45 & 24 & 46.8 & 32 & 17 & 40 & 64 & 34 & 19 & 53 & 82 \\
\hline Producción & 19 & & 8 & 5 & 29 & & 58 & 16 & 14.7 & 5 & 30 & 18 & 14 & 18 & 14 & 21 & 75 \\
\hline Tamaulipas & 100 & 3 & 4 & 11 & 12 & 4 & 62 & 72 & 56.8 & 84 & 41 & 76 & 80 & 57 & 13 & 69 & 52 \\
\hline Escuela digna & 41 & 2 & 2 & 9 & 10 & 1 & 75 & 84 & 22.7 & 92 & J & 83 & 70 & 73 & 17 & 86 & 63 \\
\hline Agua/ drenaje & 23 & & 7 & 9 & 21 & 4 & 58 & 79 & 99.3 & 94 & 46 & 76 & 95 & 49 & 4 & 70 & 43 \\
\hline Electrificación & 8 & 20 & & 10 & 10 & & 55 & 45 & 95.2 & 75 & 42 & 70 & 100 & 40 & 12 & 45 & 33 \\
\hline Infraestructura & 21 & 2 & 6 & 18 & 10 & 12 & 49 & 69 & 80.6 & 80 & 40 & 84 & 80 & 57 & 11 & 61 & 37 \\
\hline Producción & 3 & & & & & & 75 & 37 & 7.2 & 50 & 46 & 50 & 75 & 25 & 50 & 38 & 67 \\
\hline
\end{tabular}

Código: TR = Tasa de respuesta; $m \$=$ miles de pesos.

NотA: La aportación comunitaria se refiere al porcentaje financiado por la comunidad al costo total de la obra.

Fuente: La misma del cuadro anterior. 
los procesos de selección. Si bien la formación de un Comité se podía dar sólo por iniciativa de los miembros de una comunidad, en cual quier caso al final todos debían ser reconocidos (o validados) por el Delegado del Programa o uno de sus representantes (Consejo Consultivo, 1994:65). Además de esta prerrogativa, los datos (cuadro 5) demuestran quemuy pocos tuvieron su origen en las propias comunidades (29\%); en general, más de la mitad de ellos (57\%) seconstituyeron por la acción directa de los Promotores deSolidaridad. Aunquela capacidad deintermediación delos responsables regionales del Pronasol en teoría no debía generar distorsiones en la identificación de la población objetivo, en la práctica la inexistencia de criterios rigurosos para la selección les otorgó facultades de facto para canalizar a sectores sociales no prioritarios. Por lo queya sabemos, los errores deselección cometidos en las entidades estudiadas se ubicaron, según los niveles de ingreso familiar, entre 58 y $73 \%$ del total de beneficiarios y queen el peor de los casos (en Tamaulipas) los intermediarios directos del programa tuvieron una mayor presencia como promotores de la organización vecinal. Con estos resultados sólo quedarían por despejar al gunas dudas sobresi tales desaciertos tuvieron su origen en motivaciones políticas no legítimas o contrarias a los procesos de democratización.

Al abordar el problema con la información proporcionada por los miembros delas mesas directivas de los Comités (cuadros 5, 6y 7) y los beneficiarios (cuadros 2 y 8 ) surge una serie de elementos que nos impiden aceptar en los térmi nos planteados las hipótesis quesuponen la presencia de intereses políticos en la actuación del Pronasol. En lo referente al proceso deformación de los Comités destacan dos hechos: en primer lugar, la participación de los partidos políticos fue insignificante como promotores de la organización social entre las comunidades beneficiadas (cuadro 5); y, en segund o térmi no, la abrumadora intervención de los funcionarios gubernamentales en el origen delos Comités, en la gran mayoría de los casos no se tradujo en imposiciones al momento de seleccionar a sus representantes (cuadros 2 y 5). En este nivel, los resultados indican que los Comités y sus dirigentes emanaron, salvo en muy 
pocas ocasiones, de decisiones democráticas tomadas por las propias comunidades.

Con lo anterior se podría argumentar que no hay evidencias para fincar responsabilidades a los intermediaros del programa sobre el perfil político de las organizaciones y de los líderes que emergieron; sin embargo, esta afirmación resulta cierta sólo en partesi consideramos que su influencia pudo haberse infiltrado previamente en el momento de la selección. Entre las características asociadas al perfil político delas comunidades beneficiadas sobresale queen casi dos tercios deellas ya existían líderes comunitarios antes de la constitución de los Comités, de los cuales casi la mitad pertenecían a al gún tipo deorganización sindical o partidista (cuadro 6). La creación delos Comités en estemedio social tuvo al gunas implicaciones en la recomposición del tablero político local, sobre todo si setoma en cuenta queal rededor dela mitad delos antiguos líderes fue desplazada por nuevos dirigentes; no obstante, estos procesos de sustitución en su mayoría no se generaron en condiciones derechazo social o por imposiciones externas. Por otro lado, en las comuni dades donde los antiguos líderes formaban partede alguna organización, en una gran proporción la instauración de los Comi tés no implicó la desaparición o el desplazamiento detales agrupaciones ni tampoco derivó en conflictos intracomunitarios; por el contrario, los datos sugieren que en estos casos los Comités tendieron a estructurarsecon el entorno organizativo preexistente entablando relaciones decooperación.

En la perspectiva de las hipótesis que suponían la existencia de una estrategia deliberada tendientea reforzar, renovar o modificar las bases del corporativismo tradicional, los datos no aportan elementos suficientes para confirmar que tales propósitos se hayan perseguido demanera sistemática. Si dividimos a las comunidades en función desus antecedentes deliderazgo, resultaría aventurado concluir, en aquellos casos dondeno había líderes (38\%) o cuando loshabía no tenían víncul os con al gún tipo deorganización (28.6\%), quel os nuevos dirigentes o los que fueron ratificados por los beneficiarios hayan aceptado integrarse a la estructura territorial del PRI. En lo queserefiereal tercer subconjunto, es decir, en los lugares 
dondehabía líderesintegradosa algún tipo deorganización (33.4\%), cabe la posibilidad de que sí se hayan desplazado a dirigentes agrupados en organizaciones independientes o que la intervención de Solidaridad haya privilegiado a aquellos que ya pertenecían a la estructura corporativa del PRI; sin embargo, aún concediendo que estos cursos de acción hubieran acontecido en todos los casos, tampoco sería aceptable afirmar que se hayan inducido medianteprocedimientos antidemocráticos.

Tomando en cuenta la importancia rel ativa de cada subconjunto y la forma como se distribuyen al interior delas entidades, resultaría más aproximado atribuir como causas de los errores cometidos en la selección delos beneficiarios a preferencias detipo económico por parte de los intermediarios del programa que a imposiciones deliberadas para fortalecer al gún tipo decorporativismo, tradicional o de nuevo cuño. Pero aún en este último caso, la evidencia apunta que no puede ser calificado como desviación otorgar apoyos públicos a comunidades quemanifiesten tendencias políticas, cual quiera que éstas sean, cuando la situación económica de las familias corresponde con el perfil dela pobreza. Ponderando ambos aspectos en los contextos investigados se desprende que los desaciertos fueron superiores en Tamaulipas, cuyas comunidades beneficiadas (cuadro 6) y dirigentes de los Comités (cuadro 7) tenían menos antecedentes devínculos con al guna forma deorganización, corporativa o no; en cambio, en los otros dos estados donde el programa obtuvo un mejor desempeño en la selección, los precedentes organizativos perfilan una mayor conexión con estructuras de tipo corporativo, sobresaliendo el caso de Durango por el al to grado de participación de los principales miembros de las mesas directivas en agrupaciones ligadas al PRI, la mayor parte provenientes del sector rural.

Demanera complementaria a estas observaciones, podemos advertir también desde el punto de vista de los beneficiarios y del resto dela población (cuadro 8) el predominio delas opiniones que rehúsan identificar el origen y funcionamiento del Pronasol con propósitos electorales o con políticas discriminatorias en función de preferencias partidistas. Por el contrario, lo que resalta en las 
CuAdro 5. Proceso de formación de los Comités de Solidaridad (\%).

\begin{tabular}{|c|c|c|c|c|c|}
\hline \multicolumn{2}{|l|}{ Procesodeformación } & \multirow{2}{*}{$\begin{array}{r}\text { Coahuila } \\
48.0\end{array}$} & \multirow{2}{*}{$\begin{array}{r}\text { Durango } \\
59.0\end{array}$} & \multirow{2}{*}{$\begin{array}{r}\text { Tamaulipas } \\
69.0\end{array}$} & \multirow{2}{*}{$\begin{aligned} \text { Total } \\
57.0\end{aligned}$} \\
\hline Quién promovióla & Los promotores de Solidaridad & & & & \\
\hline formación del Comité: & Dirigentes deun partido político & 6.0 & 6.0 & 2.0 & 5.0 \\
\hline & Los colonos o los vecinos & 30.0 & 18.0 & 11.0 & 21.0 \\
\hline & Lasjuntas depadres & 8.0 & 8.0 & 7.0 & 8.0 \\
\hline & Las autoridades & 2.0 & 7.0 & 3.0 & 4.0 \\
\hline & Otros & 5.0 & 2.0 & 8.0 & 5.0 \\
\hline \multirow{7}{*}{$\begin{array}{l}\text { El Comitéseformó } \\
\text { através de: }\end{array}$} & La comunidad & 6.0 & 14.0 & 8.0 & 9.0 \\
\hline & Una asamblea & 69.0 & 66.0 & 75.0 & 70.0 \\
\hline & Ungrupo devecinos & 17.0 & 6.0 & 6.0 & 11.0 \\
\hline & Nombramiento por promotores deSolidaridad & 5.0 & 9.0 & 9.0 & 7.0 \\
\hline & Nombramiento por autoridades municipales & 0.4 & 3.0 & 0.5 & 1.0 \\
\hline & Nombramiento por autoridades estatales & 0.0 & 1.0 & 0.0 & 0.2 \\
\hline & Otro & 1.0 & 2.0 & 1.0 & 1.0 \\
\hline \multirow{4}{*}{$\begin{array}{l}\text { Cómo sedecidieron } \\
\text { los cargos en el Comité: }\end{array}$} & Acuerdo deasamblea & 83.0 & 91.0 & 84.0 & 85.0 \\
\hline & Acuerdo delos miembros del Comité & 8.0 & 5.0 & 5.0 & 6.0 \\
\hline & Recomendación depromotores & 6.0 & 2.0 & 8.0 & 6.0 \\
\hline & Otro & 3.0 & 2.0 & 2.0 & 3.0 \\
\hline
\end{tabular}

Fuente: La misma del cuadro 3. 
CuAdro 6. Antecedentes de liderazgos en las comunidades y su relación con los Comités (\%).

\begin{tabular}{|c|c|c|c|c|c|}
\hline \multicolumn{2}{|c|}{ Antecedentes deorganización y tipos derelaciones } & Coahuila & Durango & Tamaulipas & Total \\
\hline \multicolumn{2}{|c|}{$\begin{array}{l}\text { Antes dela formación del Comitéexistía al guna persona o grupo } \\
\text { queactuara para quelas necesidades de la comunidad fueran atendidas (Sí) }\end{array}$} & 62.6 & 59.4 & 63.5 & 62.0 \\
\hline \multicolumn{2}{|c|}{ Esa persona o grupo formaba parte de una organización (Sí) } & 57.4 & 67.2 & 38.4 & 53.9 \\
\hline $\begin{array}{l}\text { A quétipo deorganización } \\
\text { perteneća: } \\
\text { (Respuestas excluyentes) }\end{array}$ & $\begin{array}{l}\text { A un sindicato } \\
\text { A una organización decolonos } \\
\text { A un partido político } \\
\text { A una organización campesina } \\
\text { A otro tipo de organización }\end{array}$ & $\begin{array}{r}7.8 \\
34.5 \\
37.9 \\
3.4 \\
13.8\end{array}$ & $\begin{array}{r}2.4 \\
4.9 \\
26.8 \\
51.2 \\
11.0\end{array}$ & $\begin{array}{l}10.3 \\
29.3 \\
13.8 \\
24.1 \\
20.7\end{array}$ & $\begin{array}{r}6.6 \\
23.8 \\
28.9 \\
23.4 \\
14.5\end{array}$ \\
\hline \multicolumn{2}{|c|}{ Siguevigenteesa organización (Sí) } & 73.3 & 78.0 & 72.4 & 74.6 \\
\hline \multicolumn{2}{|l|}{$\begin{array}{l}\text { En estemomento } \\
\text { esa organización: }\end{array}$} & $\begin{array}{l}80.0 \\
11.8\end{array}$ & $\begin{array}{r}80.3 \\
3.0\end{array}$ & $\begin{array}{l}64.3 \\
14.3\end{array}$ & $\begin{array}{r}76.7 \\
9.3\end{array}$ \\
\hline \multicolumn{2}{|c|}{ Los antiguos líderes forman partedel Comité(No) } & 50.0 & 46.7 & 55.7 & 51.0 \\
\hline $\begin{array}{l}\text { Por quérazón no forman } \\
\text { partedel Comité: } \\
\text { (Respuestas excluyentes) }\end{array}$ & $\begin{array}{l}\text { No estaban cuando seformó } \\
\text { No lesinteresó } \\
\text { La comunidad no quiso } \\
\text { Los promotores no los aceptaron } \\
\text { Otra razón }\end{array}$ & $\begin{array}{r}12.0 \\
25.3 \\
26.5 \\
7.2 \\
28.9\end{array}$ & $\begin{array}{r}5.0 \\
32.5 \\
17.5 \\
0.0 \\
45.0\end{array}$ & $\begin{array}{r}8.2 \\
26.2 \\
23.0 \\
3.3 \\
39.3\end{array}$ & $\begin{array}{r}9.2 \\
27.2 \\
23.4 \\
4.3 \\
35.9\end{array}$ \\
\hline \multicolumn{2}{|c|}{ Comunidades según sus antecedentes deliderazgo y organización (resumen) } & 100.0 & 100.0 & 100.0 & 100.0 \\
\hline \multicolumn{2}{|l|}{ Sin antecedentes } & 37.4 & 40.6 & 36.5 & 38.0 \\
\hline \multicolumn{2}{|c|}{ Con antecedentes delíderes desvinculados deorganizaciones } & 26.7 & 19.5 & 39.1 & 28.6 \\
\hline \multicolumn{2}{|l|}{$\begin{array}{l}\text { Situación en el proceso } \\
\text { deformación delos Comités }\end{array}$} & $\begin{array}{l}50.0 \\
50.0\end{array}$ & $\begin{array}{l}37.5 \\
62.5\end{array}$ & $\begin{array}{l}55.9 \\
44.1\end{array}$ & $\begin{array}{l}52.4 \\
47.6\end{array}$ \\
\hline \multicolumn{2}{|c|}{ Con antecedentes delíderes vinculados a organizaciones } & 35.9 & 39.9 & 24.4 & 33.4 \\
\hline $\begin{array}{l}\text { Situación en el proceso } \\
\text { deformación delos Comités }\end{array}$ & $\begin{array}{l}\text { Exclusión o sustitución delíderesy organizaciones } \\
\text { Inclusión delíderes u organizaciones } \\
\text { Inclusión delíderes y organizaciones }\end{array}$ & $\begin{array}{l}33.0 \\
26.4 \\
40.6\end{array}$ & $\begin{array}{l}20.0 \\
35.5 \\
44.5\end{array}$ & $\begin{array}{l}39.1 \\
34.8 \\
26.1\end{array}$ & $\begin{array}{l}30.5 \\
30.5 \\
38.9\end{array}$ \\
\hline
\end{tabular}

Fuente: La misma del cuadro 3. 
CuAdro 7. Antecedentes de participación política delos directivos de los Comités deSolidaridad (\%).

\begin{tabular}{|c|c|c|c|c|c|c|c|c|c|c|c|c|c|c|c|c|}
\hline \multirow{2}{*}{$\begin{array}{c}\text { Cargo } \\
\text { en lamesa } \\
\text { directiva }\end{array}$} & \multirow[t]{2}{*}{ TR } & \multicolumn{2}{|c|}{ Sexo } & \multicolumn{6}{|c|}{ Perteneáa a una organización antes de formar partedel Comité } & \multicolumn{5}{|c|}{$\begin{array}{l}\text { Esaorganización pertenece } \\
\text { aalgún partido político }\end{array}$} & \multicolumn{2}{|c|}{$\begin{array}{l}\text { Sigueen esa } \\
\text { organización }\end{array}$} \\
\hline & & M & $\mathbf{F}$ & Sí & $\begin{array}{l}\text { Sindicato } \\
\text { de colonos }\end{array}$ & $\begin{array}{l}\text { Organizadión } \\
\text { político }\end{array}$ & $\begin{array}{c}\text { Partido } \\
\text { campesina }\end{array}$ & $\begin{array}{l}\text { Organización } \\
\text { la }\end{array}$ & Otras & No & PRI & PAN & PRD & Otros & Sí & No \\
\hline \multicolumn{17}{|c|}{ Total (Número promedio demiembros en una mesa directiva $=3.5$ ) } \\
\hline $\begin{array}{l}\text { Presidente } \\
\text { Secretario } \\
\text { Tesorero } \\
\text { Vocal } 1 \\
\text { Vocal } 2 \\
\text { Vocal } 3\end{array}$ & $\begin{array}{l}81 \\
77 \\
73 \\
38 \\
30 \\
21\end{array}$ & $\begin{array}{l}53 \\
43 \\
44 \\
34 \\
26 \\
27\end{array}$ & $\begin{array}{l}47 \\
57 \\
56 \\
66 \\
74 \\
73\end{array}$ & $\begin{array}{l}43 \\
34 \\
33 \\
22 \\
14 \\
18\end{array}$ & $\begin{array}{l}26 \\
31 \\
27 \\
33 \\
48 \\
50\end{array}$ & $\begin{array}{r}12 \\
6 \\
7 \\
6 \\
6 \\
7\end{array}$ & $\begin{array}{l}17 \\
19 \\
19 \\
31 \\
30 \\
23\end{array}$ & $\begin{array}{l}31 \\
34 \\
35 \\
17 \\
12 \\
10\end{array}$ & $\begin{array}{r}11 \\
17 \\
11 \\
12 \\
3 \\
7\end{array}$ & $\begin{array}{l}14 \\
13 \\
16 \\
15 \\
24 \\
20\end{array}$ & $\begin{array}{l}67 \\
67 \\
66 \\
67 \\
67 \\
67\end{array}$ & $\begin{array}{l}6 \\
7 \\
2 \\
3 \\
0 \\
3\end{array}$ & $\begin{array}{l}5 \\
5 \\
5 \\
8 \\
6 \\
3\end{array}$ & $\begin{array}{l}3 \\
4 \\
4 \\
0 \\
0 \\
0\end{array}$ & $\begin{array}{l}84 \\
86 \\
87 \\
81 \\
88 \\
83\end{array}$ & $\begin{array}{l}6 \\
3 \\
4 \\
6 \\
3 \\
7\end{array}$ \\
\hline \multicolumn{17}{|c|}{ Coahuila (Número promedio demiembros en una mesa directiva $=4.0$ ) } \\
\hline $\begin{array}{l}\text { Presidente } \\
\text { Secretario } \\
\text { Tesorero } \\
\text { Vocal } 1 \\
\text { Vocal } 2 \\
\text { Vocal } 3\end{array}$ & $\begin{array}{l}91 \\
82 \\
78 \\
56 \\
43 \\
26\end{array}$ & $\begin{array}{l}30 \\
28 \\
31 \\
31 \\
22 \\
19\end{array}$ & $\begin{array}{l}70 \\
72 \\
69 \\
69 \\
78 \\
81\end{array}$ & $\begin{array}{l}41 \\
29 \\
26 \\
22 \\
14 \\
19\end{array}$ & $\begin{array}{l}34 \\
43 \\
37 \\
41 \\
58 \\
62\end{array}$ & $\begin{array}{l}19 \\
12 \\
12 \\
10 \\
10 \\
12\end{array}$ & $\begin{array}{l}22 \\
21 \\
26 \\
33 \\
26 \\
19\end{array}$ & $\begin{array}{l}5 \\
4 \\
1 \\
0 \\
0 \\
0\end{array}$ & $\begin{array}{r}16 \\
15 \\
22 \\
13 \\
5 \\
6\end{array}$ & $\begin{array}{l}22 \\
17 \\
23 \\
18 \\
32 \\
31\end{array}$ & $\begin{array}{l}56 \\
53 \\
52 \\
56 \\
53 \\
56\end{array}$ & $\begin{array}{r}11 \\
14 \\
1 \\
5 \\
0 \\
0\end{array}$ & $\begin{array}{r}7 \\
8 \\
9 \\
10 \\
10 \\
6\end{array}$ & $\begin{array}{l}2 \\
2 \\
1 \\
0 \\
0 \\
0\end{array}$ & $\begin{array}{l}81 \\
86 \\
77 \\
77 \\
89 \\
88\end{array}$ & $\begin{array}{r}9 \\
3 \\
8 \\
10 \\
5 \\
6\end{array}$ \\
\hline \multicolumn{17}{|c|}{ Durango (N úmero promedio demiembros en una mesa directiva =2.8) } \\
\hline $\begin{array}{l}\text { Presidente } \\
\text { Secretario } \\
\text { Tesorero } \\
\text { Vocal } 1 \\
\text { Vocal } 2 \\
\text { Vocal } 3\end{array}$ & $\begin{array}{r}64 \\
73 \\
67 \\
12 \\
6 \\
4\end{array}$ & $\begin{array}{l}76 \\
68 \\
64 \\
60 \\
50 \\
56\end{array}$ & $\begin{array}{l}24 \\
32 \\
36 \\
40 \\
50 \\
44\end{array}$ & $\begin{array}{l}61 \\
56 \\
56 \\
52 \\
33 \\
33\end{array}$ & $\begin{array}{r}99 \\
10 \\
9 \\
15 \\
25 \\
33\end{array}$ & $\begin{array}{l}4 \\
4 \\
4 \\
0 \\
0 \\
0\end{array}$ & $\begin{array}{r}13 \\
14 \\
14 \\
8 \\
25 \\
33\end{array}$ & $\begin{array}{l}72 \\
70 \\
68 \\
69 \\
50 \\
33\end{array}$ & $\begin{array}{l}2 \\
2 \\
2 \\
8 \\
0 \\
0\end{array}$ & $\begin{array}{l}0 \\
1 \\
1 \\
0 \\
0 \\
0\end{array}$ & $\begin{array}{r}89 \\
85 \\
82 \\
92 \\
100 \\
100\end{array}$ & $\begin{array}{l}0 \\
0 \\
0 \\
0 \\
0 \\
0\end{array}$ & $\begin{array}{l}5 \\
5 \\
5 \\
8 \\
0 \\
0\end{array}$ & $\begin{array}{l}4 \\
6 \\
7 \\
0 \\
0 \\
0\end{array}$ & $\begin{array}{l}93 \\
92 \\
95 \\
92 \\
75 \\
67\end{array}$ & $\begin{array}{l}4 \\
2 \\
3 \\
0 \\
0 \\
0\end{array}$ \\
\hline \multicolumn{17}{|c|}{ Tamaulipas (N úmero promedio de miembros en una mesa directiva =3.4) } \\
\hline $\begin{array}{l}\text { Presidente } \\
\text { Secretario } \\
\text { Tesorero } \\
\text { Vocal } 1 \\
\text { Vocal } 2 \\
\text { Vocal } 3\end{array}$ & $\begin{array}{l}80 \\
74 \\
73 \\
35 \\
34 \\
30\end{array}$ & $\begin{array}{l}56 \\
44 \\
46 \\
32 \\
30 \\
32\end{array}$ & $\begin{array}{l}44 \\
56 \\
54 \\
68 \\
70 \\
68\end{array}$ & $\begin{array}{l}35 \\
22 \\
24 \\
14 \\
12 \\
15\end{array}$ & $\begin{array}{l}32 \\
51 \\
46 \\
25 \\
40 \\
36\end{array}$ & $\begin{array}{l}9 \\
3 \\
2 \\
0 \\
0 \\
0\end{array}$ & $\begin{array}{l}15 \\
23 \\
17 \\
50 \\
40 \\
27\end{array}$ & $\begin{array}{l}29 \\
18 \\
22 \\
17 \\
20 \\
18\end{array}$ & $\begin{array}{r}10 \\
6 \\
12 \\
8 \\
0 \\
9\end{array}$ & $\begin{array}{r}18 \\
31 \\
34 \\
25 \\
20 \\
9\end{array}$ & $\begin{array}{l}62 \\
56 \\
58 \\
75 \\
80 \\
73\end{array}$ & $\begin{array}{r}4 \\
10 \\
5 \\
0 \\
0 \\
9\end{array}$ & $\begin{array}{l}0 \\
0 \\
0 \\
0 \\
0 \\
0\end{array}$ & $\begin{array}{l}1 \\
0 \\
0 \\
0 \\
0 \\
0\end{array}$ & $\begin{array}{l}82 \\
79 \\
88 \\
83 \\
90 \\
82\end{array}$ & $\begin{array}{l}3 \\
3 \\
2 \\
0 \\
0 \\
0\end{array}$ \\
\hline
\end{tabular}

Fuente: La misma del cuadro 3. 
percepciones deestos dos subconjuntos sociales es la imagen de un programa preocupado por el bienestar y la participación delagente, orientado a promover la unión y a recuperar la confianza del pueblo en el gobierno, responsableen la medida en quecumplecon lo prometido y con el cual vale la pena continuar trabajando. Lo expuesto hasta este momento indica que no hay elementos suficientes para concluir queSolidaridad intervino demanera sistemática con el fin de ampliar, recuperar o consolidar las relaciones gobierno-sociedad empleando esquemas decontrol corporativo, ni tampoco para afirmar quesehayan utilizado los recursos públicos de manera generalizada con fines electorales específicos. La evidencia en su conjunto ratifica la hipótesis al ternativa que planteamos en el párrafo anterior: la mayor partedelos errores cometidos en los procesos de selección tienen su origen en el orden de preferencia que los intermediarios del programa concedieron a sectores sociales no prioritarios en función desu situación económica.

Esta conclusión no significa que la actuación pública haya estado desprovista de motivaciones políticas, principalmente si aceptamos que las preferencias de tipo económico pudieran estar dirigidas a restablecer nexos con amplios segmentos de la clase media norteña, cuyo peso electoral es significativo y que en los últimos tiempos habían venido mostrando tendencias contrarias al PRI. Decomprobarse esteescenario, estaríamosigual menteante la presencia de desviaciones gubernamentales originadas en objetivos derentabilidad política, pero fundados en una lógica distinta a la corporativa, es decir, más próximos a una visión liberal dela política que pretende recuperar legitimidad en sectores sociales estratégicos cada vez más plurales y ciudadanos. Desconocemos en quémedida los agentes políticos locales ligados al PRI lograron beneficiarse con esta probabl e estrategia o de qué manera afectó a los contendientes dela oposición en los procesos electorales; lo que sí parece confirmarse, como lo muestra la información, es que el presidente Sal inas adquirió una presencia central en las percepciones social es como creador del programa y muy probablemente fue quien capitalizó gran partedelos rendimientos políticos alcanzados con su instrumentación (Dion, 2000). ${ }^{2}$ 
CuAdRo 8. Percepciones acerca del origen y los propósitos que suponen la creación o existencia del Pronasol (\% sólo de respuestas afirmativas a menos que se indique lo contrario).

\begin{tabular}{|c|c|c|c|c|c|c|}
\hline \multicolumn{2}{|c|}{ El programadeSolidaridad... } & \multicolumn{4}{|c|}{ Beneficiarios } & \multirow{2}{*}{ Nobeneficiarios } \\
\hline & & Coahuila & Durango & Tamaulipas & Total & \\
\hline $\begin{array}{l}\text { Fuecreado por: } \\
\text { (Respuestasno excluyentes) }\end{array}$ & $\begin{array}{l}\text { el Presidente } \\
\text { el Gobierno } \\
\text { el PRI } \\
\text { la Comunidad } \\
\text { la Iniciativa Privada } \\
\text { la Oposición }\end{array}$ & $\begin{array}{l}75.1 \\
70.0 \\
45.0 \\
39.8 \\
20.0 \\
12.0\end{array}$ & $\begin{array}{r}82.3 \\
56.1 \\
26.0 \\
16.7 \\
10.1 \\
5.1\end{array}$ & $\begin{array}{r}85.8 \\
72.9 \\
44.9 \\
27.8 \\
10.7 \\
5.1\end{array}$ & $\begin{array}{r}80.8 \\
67.1 \\
39.6 \\
29.1 \\
14.0 \\
7.7\end{array}$ & $\begin{array}{r}80.0 \\
69.7 \\
39.0 \\
34.3 \\
13.5 \\
5.0\end{array}$ \\
\hline $\begin{array}{l}\text { Lo queleimporta es el bien } \\
\text { Fomenta la participación de } \\
\text { Beneficia a las comunidades } \\
\text { Con programas como Solida } \\
\text { Representa la unión de gobie } \\
\text { Usted estaría dispuesto a pa } \\
\text { Secreó para recuperar la con }\end{array}$ & $\begin{array}{l}\text { liay la comunidad } \\
\text { de partido } \\
\text { rno está cumpliendo lo que prometió } \\
\text { dad para mejorar } \\
\text { uras acciones del Programa } \\
\text { blo en el gobierno }\end{array}$ & $\begin{array}{l}89.6 \\
89.9 \\
84.4 \\
78.9 \\
81.6 \\
83.5 \\
78.8\end{array}$ & $\begin{array}{l}94.9 \\
89.9 \\
88.5 \\
85.9 \\
79.0 \\
86.0 \\
69.4\end{array}$ & $\begin{array}{l}87.5 \\
86.3 \\
82.2 \\
73.2 \\
74.0 \\
64.1 \\
76.8\end{array}$ & $\begin{array}{l}90.4 \\
88.4 \\
84.8 \\
78.9 \\
78.3 \\
77.5 \\
75.4\end{array}$ & $\begin{array}{l}90.0 \\
88.0 \\
81.6 \\
73.7 \\
80.0 \\
69.7 \\
79.0\end{array}$ \\
\hline $\begin{array}{l}\text { Hace quelas comunidades } p \\
\text { Hafallado en el cumplimien } \\
\text { Con programas como Solida } \\
\text { Rinde cuentas claras a las co } \\
\text { Imponesus planes a las com }\end{array}$ & $\begin{array}{l}\text { vortedelas obras (No) } \\
\text { unciono) } \\
\text { uncionarios más honestos }\end{array}$ & $\begin{array}{l}68.2 \\
60.7 \\
58.1 \\
61.2 \\
51.4\end{array}$ & $\begin{array}{l}75.6 \\
61.7 \\
63.0 \\
57.9 \\
59.6\end{array}$ & $\begin{array}{l}73.0 \\
67.9 \\
56.5 \\
55.6 \\
57.7\end{array}$ & $\begin{array}{l}72.0 \\
63.5 \\
59.0 \\
58.3 \\
55.9\end{array}$ & $\begin{array}{l}64.2 \\
51.6 \\
59.4 \\
54.9 \\
50.5\end{array}$ \\
\hline $\begin{array}{l}\text { Fuecreado para combatir el } \\
\text { Fuecreado para apoyar al PF } \\
\text { Lo quebusca es hacerleprop } \\
\text { Secreó para detener la oposi } \\
\text { Promuevelíderes queleconv } \\
\text { anipula a la gente para quev }\end{array}$ & $\begin{array}{l}\text { nes } \\
\text { ierno }\end{array}$ & $\begin{array}{l}52.0 \\
43.8 \\
38.0 \\
39.5 \\
40.0 \\
30.6\end{array}$ & $\begin{array}{l}63.9 \\
25.7 \\
33.1 \\
28.7 \\
25.2 \\
14.2\end{array}$ & $\begin{array}{l}52.5 \\
39.5 \\
35.6 \\
33.0 \\
33.3 \\
19.1\end{array}$ & $\begin{array}{l}55.5 \\
37.2 \\
35.8 \\
34.2 \\
33.5 \\
22.0\end{array}$ & $\begin{array}{r}58.6 \\
36.8 \\
39.8 \\
33.2 \\
38.0 \\
23.9\end{array}$ \\
\hline
\end{tabular}

Fuente:El Colef. Proyecto: Impacto social del Programa Nacional de Solidaridad en los estados de Coahuila, Durango y Tamaulipas, tercer trimestre de 1993. "Cédula para la identificación de beneficiarios" y "Cuestionario a beneficiarios". 


\section{Bibliografía}

Bartra, Armando. 1992. “Más sobre Pronasol”, en Consejo Consultivo del Pronasol, D esigualdad y democracia, El N acional, México.

Consejo Consultivo del Pronasol. 1994. EI Programa N acional deSolidaridad. U na visión dela modernización de M éxico, FCE, México.

Contreras, Óscar y otros. 1993. “Metodología para la evaluación externa del Programa Nacional de Solidaridad", El Colef, México, mimeo.

Dion, Michelle. 2000. “La economía política del gasto social: el Programa deSolidaridad de M éxico, 1988-1994", en E studios Sociológicos, vol. XVIII, núm. 53, mayo-agosto.

Dresser, Dense. 1994. "Pronasol y política: combate a la pobreza como fórmula degobernabilidad", en Félix Vélez (comp.), La pobreza en M éxico. Causas y políticas para combatirla, ITAM-FCE, México.

Ordóñez, Gerardo. 1997. "México en el combate a la pobreza”, en Revista Ciudades, Año 9, núm. 36, octubre-diciembre, RIU, México.

Provencio, Enrique. 1992. “La incierta relación entre desarrollo y democracia”, en Consejo Consultivo del Pronasol, D esigualdad y democracia, El N acional, México.

Artículo recibido en febrero de 2004. Artículo aprobado en abril de 2004.

2 Dion comparte la idea de que uno de los propósitos de Solidaridad "era aumentar la popularidad de Salinas", pero también afirma que aunque la distribución de sus recursos se hizo con base en criterios políticos, no tuvo la capacidad para influir "en los resultados de las elecciones presidenciales de 1994" (2000: 338 y 360). 